\title{
CONSTRUCCIÓN DE UN MICROSCOPIO PERFILOMETRICO POR INTERFEROMETRÍA DE LUZ BLANCA
}

\section{CONSTRUCTION OF A PERFILOMETRIC MICROSCOPE USING WHITE LIGHT INTERFEROMETRY}

\author{
Fis. Juan A. Sanchez-Daza, Doc. Martha Lucía Molina-Prado, Doc. Néstor. A. \\ Arias-Hernández
}

Universidad de Pamplona, Grupo de Investigación de Óptica Moderna. Ciudadela Universitaria, Pamplona, Norte de Santander, Colombia. Tel.: (+577) 5685303. E-mail: \{juanalbert.21, arias.her.nestor, molinaprado\}@ gmail.com

\begin{abstract}
Resumen: En este trabajo se presenta el diseño y construcción de un microscopio perfilométrico por interferometría de luz blanca, que permite el levantamiento topográfico de objetos con resolución nanométrica. Por medio de un escaneo axial del objeto, se adquiere un conjunto de imágenes con franjas interferométricas deformadas las cuales dan información de la rugosidad del objeto. El principio del sistema está basado en la localización del pico de coherencia en cada posición $I_{m}(i, j)$ del conjunto de $m$ imágenes adquiridas, en donde la posición $z(m)$ del pico de coherencia cambia de acuerdo a la topografía del objeto. Se presenta una serie de reconstrucciones tridimensionales de varios objetos a resolución nanométrica, obtenidas de manera automática con la ayuda de una interface gráfica elaborada en Matlab, que permite el control de los dispositivos, procesado digital de las imágenes y el cálculo de la topografía del objeto.
\end{abstract}

Palabras claves: Interferometría de luz blanca, Mirau, Sistema interferómetro Vertical.

\begin{abstract}
Design and construction of a perfilometric microscope by white light interferometry is presented. This perfilometric microscope allows the topographic lifting of objects with nanometric resolution. By means of an axial scanning of the object, a set of images is acquired. The set of images they are obtained using a system of deformed interferometric fringes, which give information of the roughness of the object. The principle of the system it is based on the location of the coherence peak in each position $\operatorname{Im}(i, j)$ of the set of $\mathrm{m}$ images, where the position $z(m)$ of the coherence peak changes according to the topography of the object. As results, a series of three-dimensional reconstructions of several objects at nanometric resolution is presented, obtained automatically with the help of a graphical interface elaborated in Matlab, which allow images digital processing and control of the devices.
\end{abstract}

Keywords: White light interferometry, Mirau, Vertical interferometer system.

\section{INTRODUCCIÓN}

La interferometría de luz blanca es una técnica muy conocida en el estudio de topografías de objetos del orden nanométrico. Davidson (1987) utilizó la técnica de interferometría de luz policromática para medir el perfil de microcomponentes en los circuitos integrados. Lee y Strand, (1990) demostraron que la resolución lateral podría mejorarse con interferometría de luz policromática o luz blanca en comparación con la del microscopio convencional. En los últimos años se han publicado muchas más aplicaciones de la interferometría luz blanca (Deck y Groot, 1994; 
Sandoz, 1999). Este trabajo describe el diseño y construcción de un sistema microscópico con un objetivo interferométrico tipo Mirau de 10X, que permite extraer la información tridimensional de la muestra observada, la cual, se obtiene a partir de las imágenes adquiridas. Para calcular el pico de coherencia se implementa varios algoritmos como son: el cálculo del mínimo del interferograma, el cálculo del máximo de la envolvente (Takeda, 1982; Larkin, 1996) y la localización del centro de interferograma mediante el análisis de la fase por transformada de Fourier (Sandoz 1996; Gasvik, 2002). (a)

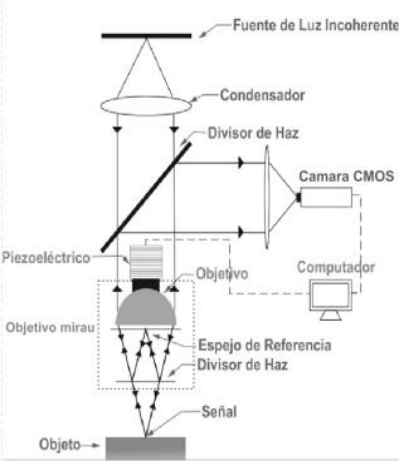

(b)

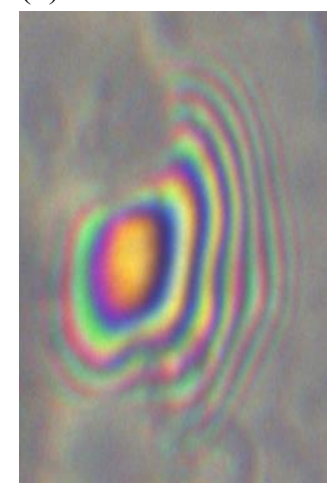

Fig. 1. (a) Esquema de sistema interferométrico tipo Mirau. (b) imagen interferométrica registrada por la cámara para cierta región de una esfera metalizada.

Una imagen interferométrica está compuesta por un sistema de franjas que contiene información de intensidad y de fase asociados a la muestra (objeto) (Fig. 1(b)). El objetivo interferométrico tipo Mirau, contiene en su interior un interferómetro que está compuesto por un divisor de haz entre el objetivo y la muestra (Fig. 1(a)), y un espejo de referencia sobre el centro de su cara frontal. El funcionamiento del objetivo interferométrico es similar al de un interferómetro tipo Michelson, con la diferencia que los dos brazos por donde la luz realiza su recorrido están en un mismo eje, por lo tanto, cuando el haz luminoso atraviesa el objetivo, el divisor de haz o lámina separatríz divide éste en dos: uno que se transmite hacia la muestra y el otro que es reflejado. El haz reflejado va al espejo de referencia, regresa a la lámina separatríz y se superpone con el reflejado por el objeto. Así, la correlación de los dos haces es vista por el sensor CMOS. El contacto óptico se obtiene cuando la diferencia de camino óptico entre el haz-espejo de referencia y el haz-objeto es cero. En este trabajo se detalla el microscopio construido, un esquema de funcionamiento del mismo, una descripción de los algoritmos de detección del pico de coherencia implementados, la interface de control de la cámara digital y el sistema de traslación piezoeléctrica. De igual manera se presenta una calibración de sistema en precisión, resolución y rango de trabajo, y se da a conocer las ventajas y desventajas del uso de esta técnica.

\section{ANALISIS DE FRANJAS DE INTERFERENCIA DE LUZ POLICROMATICA}

Al utilizar el sistema mostrado de la Figura 1(a)(b) con luz blanca o policromática, las reflexiones sobre la muestra, ubicada en el portaobjeto, son combinadas con las reflexiones provenientes del espejo de referencia, localizado en el objetivo Mirau. El campo eléctrico $E(t)$ que llega al detector es la superposición de la luz que proviene de la muestra y el espejo de referencia:

$$
E(t)=E_{s}(t)+E_{r}(t+\tau)
$$

Donde $E_{s}$ y $E_{r}$ son respectivamente las amplitudes ópticas del haz señal o de la muestra y del haz de referencia. Mientras que $\tau$, es el tiempo de retraso debido a la diferencia de longitud en los caminos ópticos de los dos haces. La intensidad registrada por el detector está dada por,

$$
I_{d}=\left\langle|E(t)|^{2}\right\rangle=I_{s}+I_{r}+2 \sqrt{I_{s} I_{r}} \mathbf{R} e\{\gamma(\tau)\}
$$

donde $\gamma(\tau)$ es la forma normalizada del grado complejo de coherencia mutua, que se expresa como,

$$
()=\frac{\left\langle E_{s}^{\prime}() E_{r}(t+)\right\rangle}{\sqrt{I_{s}+I_{r}}}
$$

En general, el grado complejo de coherencia mutua incluye los efectos de coherencia espacial y temporal. Para un interferómetro de amplitud como el interferómetro Mirau, la coherencia espacial se puede despreciar considerando un punto sobre la fuente incoherente espacialmente, así la coherencia mutua se reduce a la auto-coherencia o coherencia temporal, en este caso:

$$
I_{d}(\tau)=\left\langle|E(\tau)|^{2}\right\rangle=I_{s}+I_{r}+2 \sqrt{I_{s} I_{r}} \operatorname{R} e\left\{\gamma_{t c}(\tau)\right\}
$$

Donde, $\boldsymbol{R} e\left\{\gamma_{t c}(\tau)\right\}$ es la parte real del grado complejo de coherencia temporal de la fuente de luz, la cual es la forma normalizada de la función de auto-coherencia $\gamma_{11}(\tau)$,

$$
{ }_{11}()=\frac{{ }_{11}()}{{ }_{11}(0)}
$$


Donde $\Gamma_{11}=\left\langle E(t+\tau) E^{*}(\tau)\right\rangle$ y el subíndice 11, corresponde a un único punto de la fuente incoherente. El grado complejo de coherencia normalizado de la fuente de luz es dado por la trasformada de Fourier de la densidad espectral de energía de la fuente de luz. Si esta fuente de luz es policromática y tiene una densidad espectral de energía gaussiana $G(f)$ con ancho espectral $\Delta f \mathrm{y}$ frecuencia media $\bar{f}$, el grado complejo de coherencia temporal $\gamma_{11}$ estará dado por,

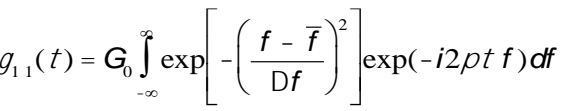

Donde $G_{0}$ es una constante. Teniendo en cuenta que $\gamma_{11}(0)=1$, se tiene que:

$$
\gamma_{t \mathrm{c}}(\tau)=\exp \left[-(\pi \tau \Delta f)^{2}\right] \exp (-i 2 \pi \bar{f} \tau)
$$

Así la ecuación (4), se puede escribir como:

$$
\begin{gathered}
I_{d}(\tau)=I_{s}+I_{r}+2 \sqrt{I_{s} I_{r}} \exp \left[-(\pi \tau \Delta f)^{2}\right] \cos (2 \pi \bar{f} \tau) \\
I_{d}=I_{0}[1+V(\tau) \cos (2 \pi \bar{f} \tau)]
\end{gathered}
$$$$
\text { o }
$$

Donde $I_{0}$ es la intensidad de fondo $\left(I_{s}+I_{r}\right)$, $V$ es la función de contraste de las franjas o envolvente del patrón de franjas observado. Esta función $V$ está dada por:

$$
V(\tau)=\left(\frac{2 \sqrt{I_{s} I_{r}}}{I_{s}+I_{r}}\right) \exp \left[-(\pi \tau \Delta f)^{2}\right]
$$



(a)

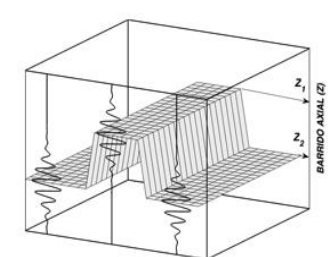

(b)
Fig. 2. (a) Perfil interferométrico de un pixel o interferograma. (b) Localización de los picos de coherencia de los inteferogramas y determinación de las medidas de alturas para tres pixeles arbitrarios.

Si en el sistema de la Figura 2(a) y (b) el origen de coordenadas es tomado sobre un punto $z$ en particular en la dirección axial, donde los dos caminos ópticos son iguales (contacto óptico) y la superficie de prueba es movida a lo largo del eje $Z$ en una serie de pasos de tamaño $\Delta Z$, entonces la intensidad sobre un punto $(x, y)$ en el plano de la imagen que corresponde a un punto del objeto $\mathrm{d}$ altura $h$, se puede escribir como:

$$
I_{d}(\tau)=I_{s}+I_{r}+2 \sqrt{I_{s} I_{r}} \gamma\left(\frac{p}{c}\right) \cos \left[\left(\frac{2 \pi}{\bar{\lambda}}\right) p+\phi_{0}\right]
$$

donde $I_{s}$ e $I_{r}$ son las intensidades de los dos haces que actúan independientemente, $\gamma\left(\frac{p}{c}\right)$ es el grado complejo de coherencia, la cual corresponde a la visibilidad de la envolvente de la franjas de interferencia, $\mathrm{y} \cos \left[\left(\frac{2 \pi}{\bar{\lambda}}\right) p+\phi_{0}\right]$ es la modulación cosenoidal donde $\bar{\lambda}$ corresponde a la longitud de onda media de la fuente, $p=2(z-h)$ es la diferencia de las longitudes de los caminos ópticos atravesados por los haces y $\phi_{0}$ es la diferencia del corrimiento de fase debido a las reflexiones sobre el divisor de haz, los espejos y posiblemente el material de prueba. La Figura 2(b), muestra las variaciones de intensidad sobre el punto de la imagen cuando el objeto es barrido axialmente. Se utiliza una cámara CMOS ubicada en el plano imagen del sistema óptico del microscopio como mecanismo de registro del interferograma, cada pixel de esta tiene la posibilidad de registrar patrones de interferencia similares a los de la Figura 2(a) en la medida que el objeto es desplazado en el eje axial $Z$, pero estos pueden tener la posición pico de coherencia en posiciones de barrido diferentes, esta diferencia de posiciones nos permite medir la forma del objeto (Fig. 2(b)).

\section{METODOS DE DETECCION DEL PICO DE COHERENCIA}

La interferometría con luz blanca es una técnica ampliamente utilizada para medidas topográficas de alta resolución axial sin contacto (Deck y Groot, 1994; Larkin, 1996; Sandoz, 1996). La técnica básicamente divide un rayo de luz de una fuente de luz blanca en dos haces separados. Un rayo es reflejado por la superficie del objeto a medirse (Brazo muestra) mientras que el otro sigue un trayecto conocido de distancia óptica constante (Brazo de referencia). Debido al ancho de banda espectral de la fuente, la longitud de coherencia es corta, y franjas de buen contraste se obtendrán únicamente cuando la diferencia de camino de los dos brazos del interferómetro sea menor a la longitud de coherencia de la fuente. Se utiliza un transductor piezoeléctrico (PZT), para variar la distancia óptica del brazo muestra del interferómetro y las variaciones de altura a través de la muestra pueden ser determinadas buscando la 
posición que tiene el PZT cuando se obtiene el máximo contraste de franjas. Este modo de operación es conocido como Modo de sensado del pico de coherencia por barrido vertical.

\subsection{Detección de máximos o mínimo del interferograma.}

La detección del máximo o mínimo es una técnica que aprovecha la propiedad de los interferogramas producidos por una fuente policromática. Según la Ecuación (9), las franjas se encuentran moduladas por una envolvente $V(\tau)$ presentando un máximo o mínimo de intensidad en puntos donde la diferencia de camino óptico es cero. Determinar la forma del objeto consiste en localizar la posición del máximo o mínimo del interferograma para cada punto del objeto, desplazando uno de los brazos del interferómetro Mirau, de tal manera, que cada punto pasará por el plano de contacto óptico en un valor de desplazamiento del portaobjeto, dependiendo de la diferencia de altura que tenga los diferentes puntos del objeto. La cámara CMOS registrará los valores de la intensidad del interferograma de tal forma, que permite almacenar el desplazamiento del portaobjeto asociado al valor de intensidad máximo o mínimo. Así se construye una matriz con las posiciones de desplazamiento del portaobjeto que brinda información de altura del objeto discretizada en un número de niveles de gris propio de la cámara utilizada. El criterio para escoger si es el máximo o el mínimo del interferograma con el que se trabajara, dependerá si las franjas de interferencia presenten un desfase con respecto al máximo de la envolvente. Se procura escoger aquella cuya franja coincida o esté más cercana al máximo o mínimo de la envolvente. En este trabajo, debido a los materiales utilizados se escogió el valor mínimo del interferograma $\min \left\{I_{m}(i, j)\right\}$.

\subsection{Máximo de la envolvente del} interferograma

El método de para detectar el pico de coherencia usando la envolvente del interferograma utiliza la Trasformada de Fourier (Gasvik, 2002). El método de la transformada de Fourier fue concebido por Takeda (1982), quien empleó el método de la transformada rápida de Fourier (FFT). Al interferograma registrado para un pixel $g(z)$ se le aplica la Transformada de Fourier obteniendo su espectro.

$$
G\left(f_{z}\right)=\mathfrak{J}\{g(z)\}=\int_{-\infty}^{\infty} g(z) e^{-2 \pi i z f_{z}} d z
$$

En el espacio de frecuencias se ubica el primer armónico que contiene la información de

frecuencias presente en el interferograma y se filtra con una ventana tipo hanning de la forma:

$$
\operatorname{Hann}\left(f_{z}\right)=\frac{1}{8}\left[1-\cos \left(2 \pi\left(\frac{f_{z}-f_{0}}{b_{f}}\right)\right)\right]
$$

De manera que,

$$
G_{f}\left(f_{z}\right)=G\left(f_{z}\right) \bullet \operatorname{Hann}\left(f_{z}\right)
$$

Donde $f_{0}$ es la frecuencia portadora.

Posteriormente se aplica la transformada inversa,

$$
g_{f}(z)=\mathfrak{J}^{-1}\left\{G_{f}\left(f_{z}\right)\right\}=\int_{f_{z}} G_{f}\left(f_{z}\right) e^{2 \pi i f_{z}} d f_{z}
$$

Finalmente se obtiene el módulo o envolvente del interferograma.

$$
\left|g_{f}(z)\right|=\sqrt{\left(\operatorname{Re}\left[g_{f}(z)\right]\right)^{2}+\left(\operatorname{Im}\left[g_{f}(z)\right]\right)^{2}}
$$

Donde, $\operatorname{Re}\left[g_{f}(z)\right]$ y $\operatorname{Im}\left[g_{f}(z)\right]$ son la parte real e imaginara de la transformada inversa respectivamente. Finalmente se calcula el máximo de dicha envolvente asociándolo al punto donde la diferencia de camino óptico es cero. Los pasos que describen este procedimiento se pueden ver en la Tabla 1 en los pasos 1, 2, 3 y 4 .

\subsection{Localización por valores de fase}

Este método (Gasvik, 2002; Arias, 2009) sigue beneficiándose de las propiedades de la Trasformada de Fourier, específicamente de la sensibilidad de la fase o también conocida como la propiedad de corrimiento,

$$
\mathfrak{I}\left\{g_{f}(z-a)\right\}=G_{f}\left(f_{z}\right) e^{-2 \pi i\left(f_{z} a\right)}
$$

Una vez recuperada la transformada de Fourier inversa, se calcula $\left|g_{f}(z)\right|$ y la fase $p(z)$ del interferograma,

$$
p(z)=\arctan \left(\frac{\operatorname{Im}\left[g_{f}(z)\right]}{\operatorname{Re}\left[g_{f}(z)\right]}\right)
$$

Con la ayuda del módulo de la Ec.16 se crea una máscara binaria para filtrar la zona de la fase asociada al interferograma o fase de trabajo. Para localizar el pico de coherencia se desenvuelve la fase con un algoritmo de unwrapping cuyo punto inicial de desenvolvimiento y el máximo de la envolvente coinciden.

Tabla 1: Description del procedimiento

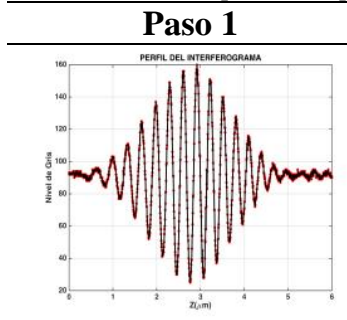

Perfil del interferograma representado por: $g(z)$

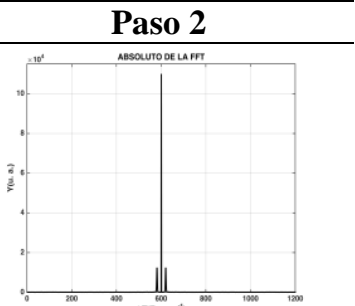

Transformada de Fourier del interferograma dada por: 


\section{$g(z)=a(z)+b(z) \cos \left[\phi(z)+2 \pi f_{0} z\right] \quad G\left(f_{z}\right)=\mathfrak{I}\{g(z)\}=\int^{\infty} g(z) e^{-2 \pi i f_{z}} d z$}

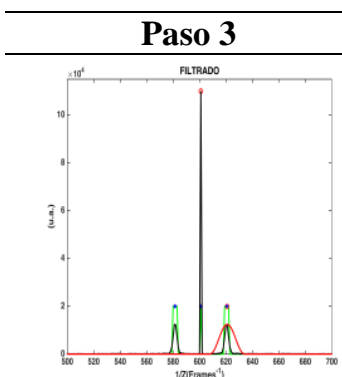

Localización y filtrado del primer armónico usando un filtro tipo hanning $G_{f}\left(f_{z}\right)=G\left(f_{z}\right) \bullet \operatorname{Hann}\left(f_{z}\right)$

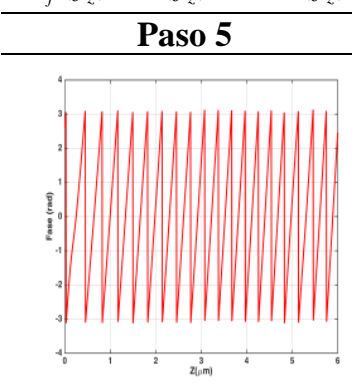

Fase de la IFFT del primer armónico

$p(z)=\arctan \left(\frac{\operatorname{Im}\left[g_{f}(z)\right]}{\operatorname{Re}\left[g_{f}(z)\right]}\right)$

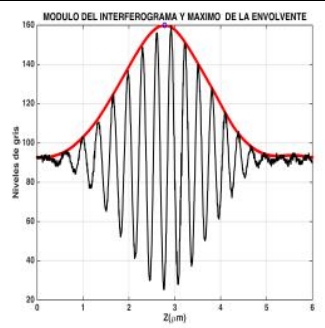

Módulo de la IFFT del primer armónico filtrado

$\left|g_{f}(z)\right|=\sqrt{\left(\operatorname{Re}\left[g_{f}(z)\right]\right)^{2}+\left(\operatorname{Im}\left[g_{f}(z)\right]\right)^{2}}$
Paso 4

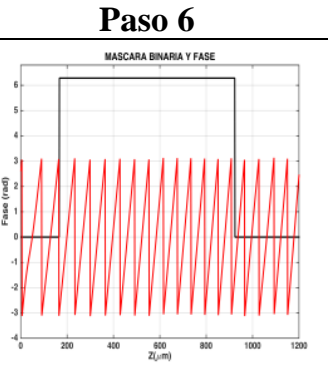

Mascara binaria obtenida a partir de la envolvente del paso 4. Permite seleccionar la fase de trabajo.

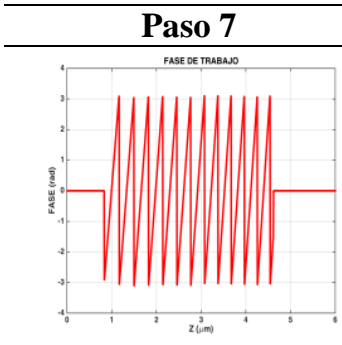

Fase de trabajo obtenida en interferograma.

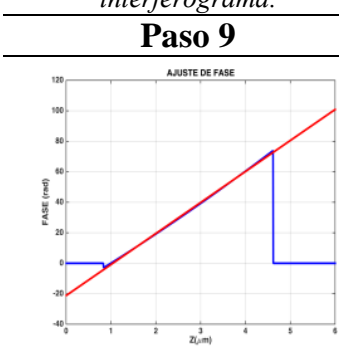

Recta de ajuste con valores de fase en zona de trabajo. $P(z)=a z+b$ el mismo dominio del

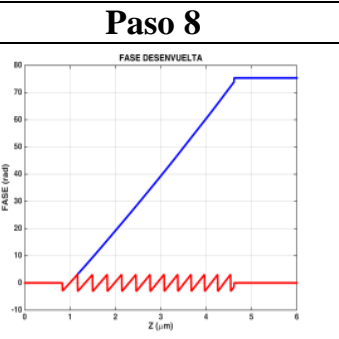

Fase Desenvuelta usando un algoritmo de Unwrapping.

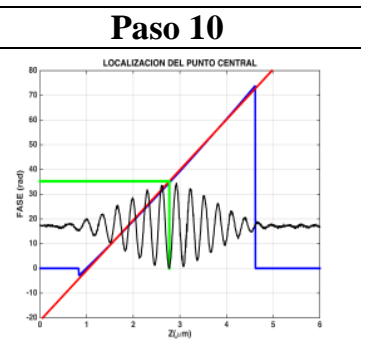

Localización del z central del interferograma para un valor de fase adecuado. $z_{i j}=\left(P_{i j}(z)-b\right) / a$
Se fija un valor de fase asociado al punto inicial $P(z)$ de desenvolvimiento para busca el valor de $z$ usando una función de ajuste por mínimos cuadrados sobre la fase desenvuelta. Los pasos que describen este procedimiento se pueden ver en la tabla 1, del paso 1 al 10.

$$
P(z)=a z+b
$$

\section{DESCRIPCION DEL MONTAJE.}

En este trabajo se construye un software para controlar el dispositivo experimental que consta de una cámara digital tipo CMOS de $752 \times 480$ pixeles $\mathrm{y}$ un objetivo interferométrico tipo Mirau 10x un sistema de traslación piezoeléctrico con resolución de $1 \mathrm{~nm}$ y rango de trabajo de $100 \mu \mathrm{m}$ y un sistema de iluminación tipo LED blanca (Fig. 3(a)). El software construido permite controlar el proceso de captura de la cámara, así como la región de interés, sincronizado con el barrido axial del piezoeléctrico. Se obtiene un numero de imágenes determinado por el paso del piezoeléctrico y la profundidad del objeto de interés (Fig. 4(a)).

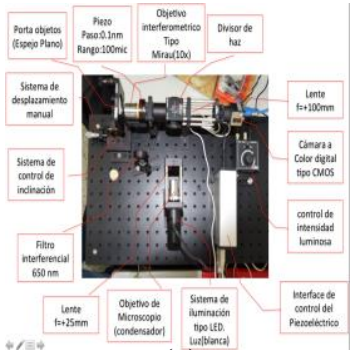

(a)

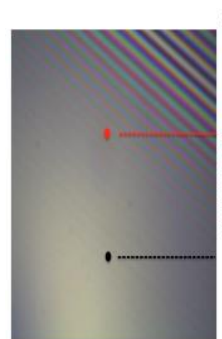

(b)

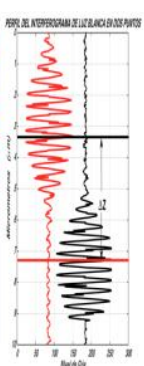

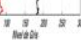

Fig. 3. (a) Montaje elaborado para la construcción del microscopio interferómetro. (b) Registro del interferograma (valor de nivel de Gris) en dos puntos del objeto a diferentes caminos ópticos, mientras el piezo realiza un barrido de 10 micrómetros, la localización de los máximos del interferograma permite establecer la diferencia de altura $\Delta z$ de los puntos seleccionados.

En este trabajo se diseñó, construyó y se realizó la puesta a punto del microscopio interferómetro, el control de los dispositivos (cámara, piezo) desde el computador (Fig. 3(a)), así mismo, la implementación de los algoritmos de detección de pico de correlación (detección del mínimo del interferograma, detección del máximo de la envolvente (Takeda, 1982; Larkin, 1996) y por detección del centro de las franjas del interferograma mediante análisis de fase por Transformada de Fourier (Sandoz, 1996)) (Fig. 4 y 5).

La interfaz permite obtener el perfil topográfico de un objeto, utilizando las tres técnicas de localización del pico de coherencia, en la Figura 5 se muestra los interferogramas en una sección vertical, para la reconstrucción de un espejo plano. Este resultado se obtuvo mediante el barrido 
vertical, con ayuda de un sistema de desplazamiento piezoeléctrico (PTZ) con paso de $5 \mathrm{~nm}$ en un rango de 6 micras.

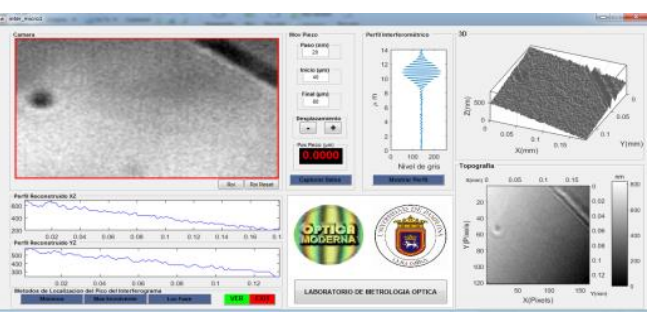

Fig. 4. Interface de control que permite la captura de los datos del interferograma y el cálculo de la topografía del objeto.

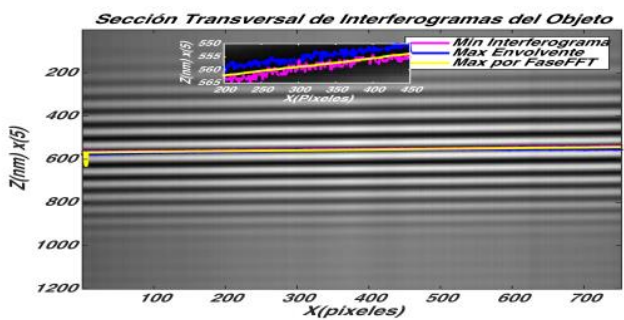

Fig. 5. Sección transversal de los interferogramas del objeto (espejo plano), en el cual se muestra los resultados del perfil del objeto por los tres métodos implementados (localización del mínimo de intensidad del interferograma, el máximo de la envolvente, y la localización del centro del interferograma por análisis de la fase por transformada de Fourier).

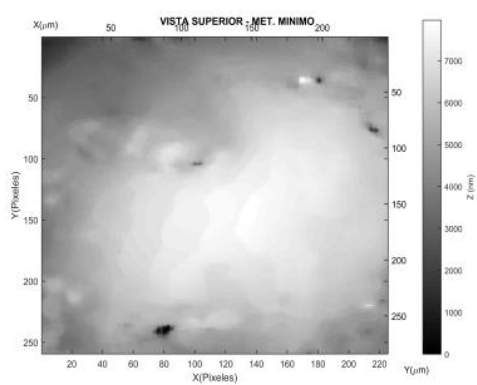

(a)

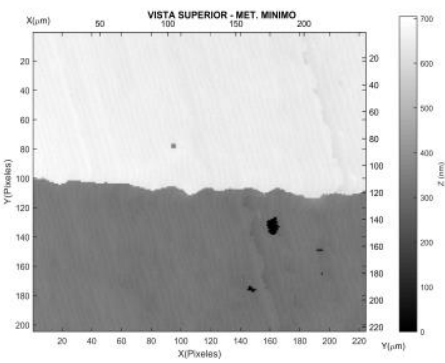

(d)

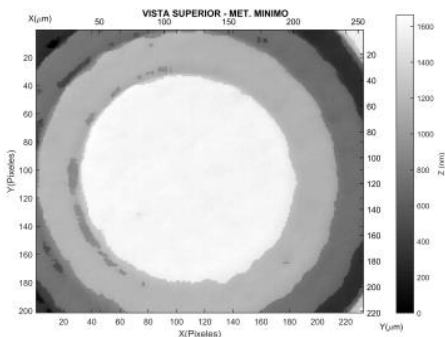

(g)

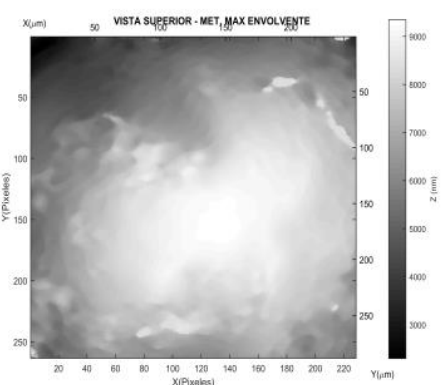

(b)

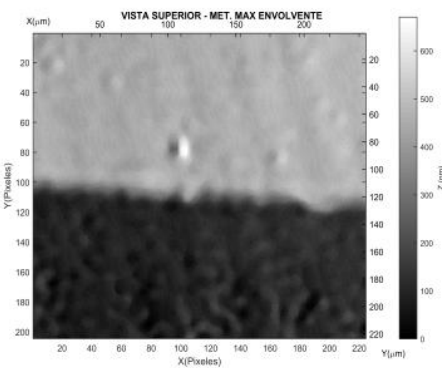

(e)

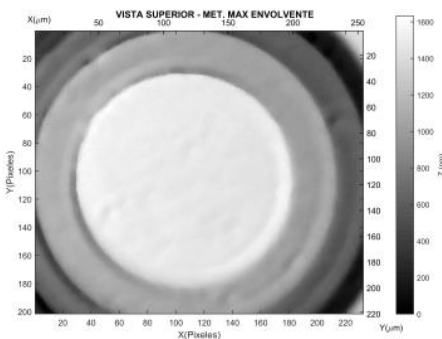

(h)

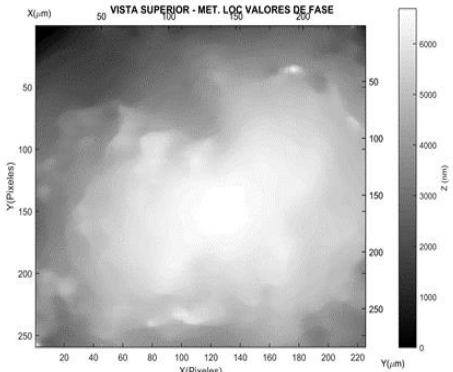

(c)

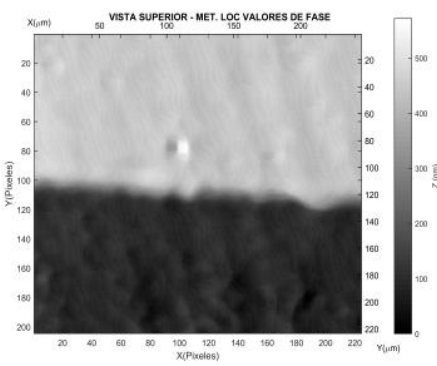

(f)

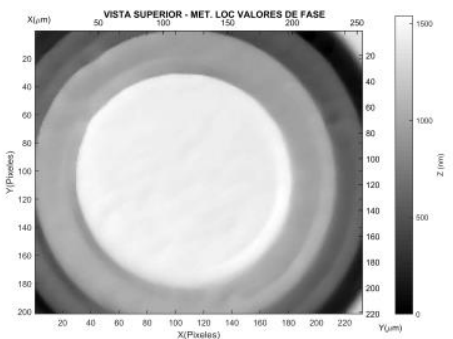

(i)

Fig. 6. Se presentan las vistas superiores obtenidas por los tres métodos: (a),(d),(g) Deteccion de Mínimos, (b),(e),(h) Máximo de la envolvente del interferoframa y (c),(f),(i) Localización por valores de Fase.

Mientras se realiza el barrido axial se almacena la matriz con los valores de intensidad y el paso del piezoeléctrico. Aplicando los algoritmos de Detección de Mínimos (MM), Máximo de la envolvente (ME) y Localización por valores de fase (MF) sobre distintas muestras, se obtiene información de su superficie. La Figura 6 (a), (b) y (c) muestra una vista superior de las reconstrucciones por los tres métodos de parte de una esfera metalizada, La Figura 6 (d), (e) y (f) muestra una vista superior de las reconstrucciones por los tres métodos de parte de una nanoestructura 


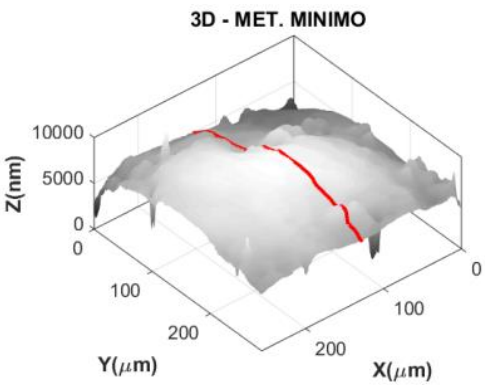

(a)

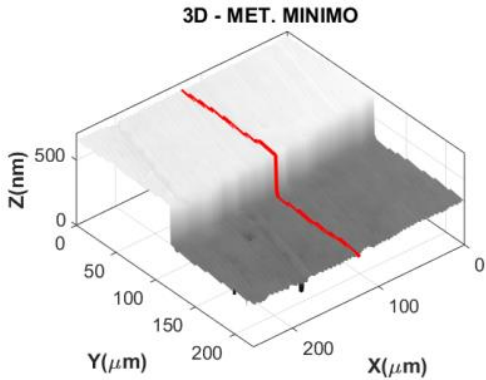

(d)

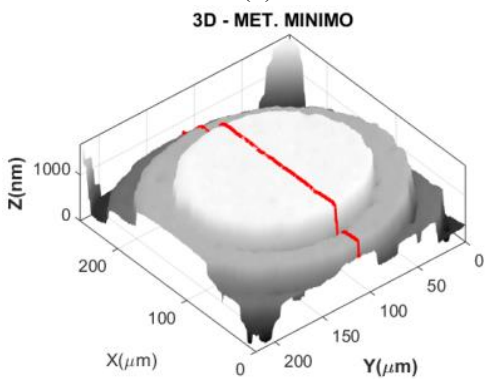

(g)

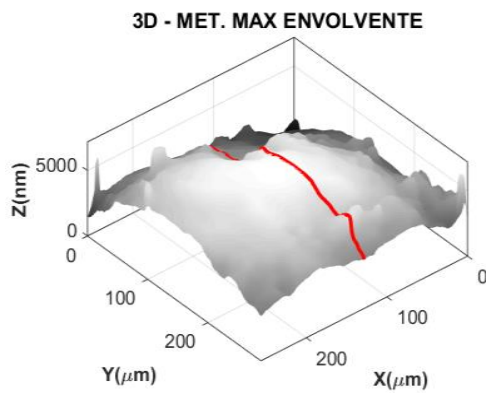

(b)

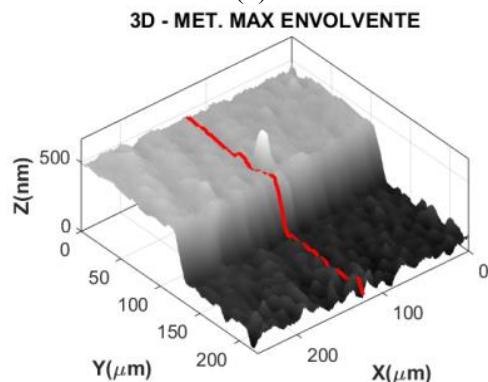

(e)

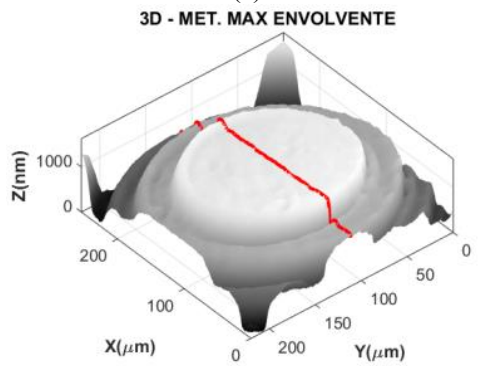

(h)

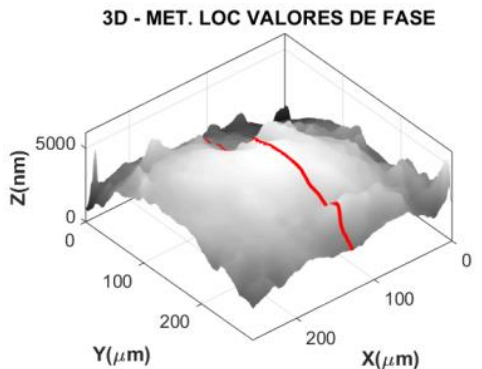

(c)

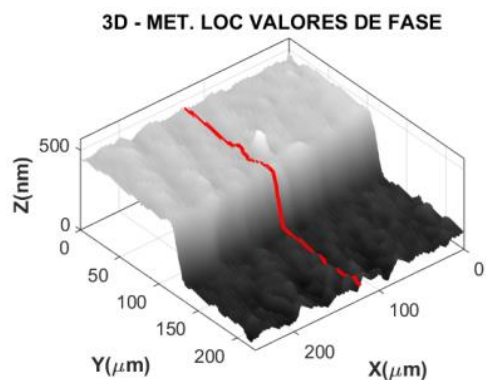

(f)

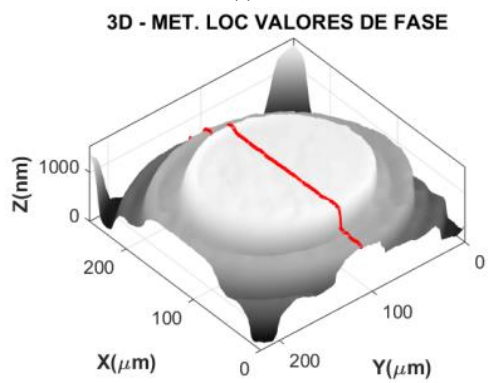

(i)

Fig. 7. Se presentan las vistas 3D obtenidas por los tres métodos: (a),(d),(g) Deteccion de Mínimos, (b),(e),(h) Máximo de la envolvente del interferoframa y (c),(f),(i) Localización por valores de Fase.

con forma de escalón y La figura 6 (g), (h) y (i) muestra una vista superior de las reconstrucciones por los tres métodos de parte de cierta región de una microlente de Fresnel. De igual forma, las Figura 7 (a), (b) y (c) muestra la vista 3D de las reconstrucciones por los tres métodos de parte de una esfera metalizada, La Figura / (d), (e) y (f) muestra la vista 3D de las reconstrucciones por los tres métodos de parte de una nanoestructura con forma de escalón y La Figura 7 (g), (h) y (i) muestra la vista $3 \mathrm{D}$ de las reconstrucciones por los tres métodos de parte de cierta región de una microlente de Fresnel. El rango de barrido axial depende de la altura de los detalles y la inclinación de la muestra, en los casos estudiados el rango fue de 4 micras a 20 micras.
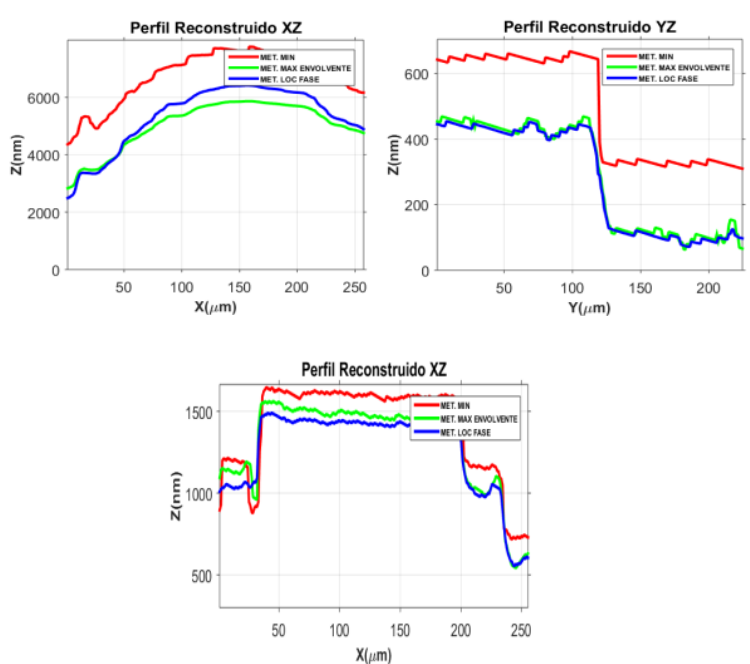

Fig. 7. Perfiles obtenidos para la reconstrucción de cada objeto: (a) esfera metalizada, (b) nanoestructura con forma de escalón y (c) microlente de Fresnel. 
Se cálculo el error para los tres métodos al reconstruir un espejo asumiéndolo perfectamente plano. El plano que representa el espejo se obtiene mediante un ajuste por mínimos cuadrados. Al calcular la desviación estándar de éste con la topografía obtenida en cada caso, permite establecer cuál de los tres métodos es la de menor error. La reconstrucción topográfica de mejor comportamiento, es la del método de Localización por valor de fase, con una desviación estándar de $9.5 \mathrm{~nm}$, seguida del método localización del método de localización del máximo de la envolvente con $14.4 \mathrm{~nm}$ y el de más bajo desempeño fue mínimo del interferograma con una desviación estándar con $23.8 \mathrm{~nm}$ de desviación estándar. Esto teniendo en cuenta que la matriz de interferogramas captadas por la cámara se registró a pasos del piezoeléctrico de $20 \mathrm{~nm}$. En la figura 7 . (a), (b) y (c) se muestran los perfiles para la esfera metalizada, el escalón respectivamente y la lente de Fresnel, obtenidos por cada método evaluado en este trabajo. Esto permite observar el comportamiento diferencial de los métodos y destacando la superioridad en calidad del método de localización de valor de fase sobre los demás.

\section{CONCLUSIONES}

Se implementó un sistema de microscopía interferencial tipo Mirau, que permite obtener la topografía de objetos con alturas del orden de los nanómetros. Este microscopio es constituido por: un piezoeléctrico con una resolución axial de $0.1 \mathrm{~nm}$ que permite obtener medidas de las alturas, una cámara CMOS de 480x752 pixeles y un objetivo interferométrico de microscopio de 10x, cuya zona de observación máxima es de $0.4 \times 0.8 \mathrm{~mm}$. Se implementó tres métodos de detección del pico de coherencia, para el cálculo de la topografía de objetos cuyas alturas son del orden de los nanómetros: de Localización por valor de fase, localización del máximo de la envolvente y búsqueda del mínimo del interferograma $\min \left\{I_{m}(i, j)\right\}$. La resolución final del sistema construido, depende del paso seleccionado y del método utilizado, de tal forma que el método de reconstrucción topográfica de resolución nanométrica de mejor comportamiento, fue el de Localización por valor de fase, con una desviación estándar de $9.5 \mathrm{~nm}$ con un paso de barrido de $20 \mathrm{~nm}$.

\section{REFERENCIAS}

Davidson, M., Kaufman, K., Mazor, I., \& Cohen, F. (1987). An application of interference microscopy to integrated circuit inspection and metrology. "Paper presented at the Integrated Circuit Metrology, Inspection, \& Process Control"

P. Sandoz, R. Escalona, V. Bonnans, and S. Dembele (1999), "From interferometry to image processing: Phase measurement vision method for high accuracy position sensing of rigid targets, Proceedings of Interferometry in Speckle Light: Theory and Applications". 421-428

P. J. Caber (1993), "Interferometric profiler for rough surfaces". Appl. Opt., 19(32):3438344.

L. Deck and P. de Groot (1994), "High-speed noncontact profiler based on scanning whitelight interferometry". Appl. Opt., 31(33):7334-7338.

P. de Groot and L. Deck (1995), "Surface profiling by analysis of white-light interferograms in the spatial frequency domain". J. of Mod. Opt., 2(42):389-401.

M. Takeda (1982), "Fourier-transform method of fringe-pattern analysis for computer-based topography and interferometry". J. Opt. Soc. Am., (72):156-160.

K. G. Larkin (1996), "Efficient nonlinear algorithm for envelope detection in white light interferometry". J. Opt. Soc. Am., 13:832843 ,

P. Sandoz (1996), “An algorithm for profilometry by white light phase shifting interferometry". J. Mod.Opt. (43):1545-1554.

Kjell J. Gasvik (2002) “Optical metrology”, Third Edition. Spectral vision AS, Trondhein, Norway.

N. Arias, M. A. Suarez, J. Meneses, Tijani Gharbi, (2009), "Medida de la Orientación, Posición y desplazamiento en el Plano de un objeto por codificación de Fase", Revista BISTUA, 2(7):70-76.

Lee, B. S., \& Strand, T. C. (1990). P"rofilometry with a coherence scanning microscope". Applied Optics, 29(26), 3784-3788. 